\title{
Investigating the Spinnability in the Dry-Jet Wet Spinning of PAN Precursor Fiber
}

\author{
Lianjiang Tan, ${ }^{1}$ Huifang Chen, ${ }^{1}$ Ding Pan, ${ }^{1}$ Ning Pan $^{2}$ \\ ${ }^{1}$ State Key Laboratory for Chemical Fibers Modification and Polymer Materials, Donghua University, \\ Shanghai 201620, People's Republic of China \\ ${ }^{2}$ BioResource and Agricultural Engineering Department, University of California, California 6561
}

Received 14 November 2007; accepted 2 January 2008

DOI 10.1002/app.28029

Published online 7 August 2008 in Wiley InterScience (www.interscience.wiley.com).

\begin{abstract}
The spinnability of a spinning solution using DMSO as the solvent was investigated for dry-jet wet spinning of PAN precursor fiber. Among many variables responsible for spinnability, the coagulating conditions, the air gap length, the nonsolvent content in spinning solution, and the spinning temperature have been viewed as the key factors, and they were investigated in this study. It was found however, unlike in the wet spinning, the spinnability in dry-jet wet spinning process was barely influenced by the coagulating conditions, likely attributable to the existence of the air gap. However, the spinnability worsened when the air gap was longer than
\end{abstract}

$30 \mathrm{~mm}$. The quality of the spinning solution deteriorated with the increasing water content in it. The spinnability improved when the spinning temperature was maintained between 60 and $72^{\circ} \mathrm{C}$ and turned down once the temperature was over $72^{\circ} \mathrm{C}$. The experimental results indicated that all the factors should be comprehensively considered to ensure good spinnability in dry-jet wet spinning process. (c) 2008 Wiley Periodicals, Inc. J Appl Polym Sci 110: 19972000, 2008

Key words: PAN precursor fiber; dry-jet wet spinning; spinnability; take-up velocity

\section{INTRODUCTION}

PAN-based carbon fiber is widely used in many industries such as aviation, aerospace, sporting goods, and construction. Properties of PAN precursor fiber are critical for yielding high-quality PANbased carbon fiber. ${ }^{1}$ In preparing PAN solutions for manufacturing PAN precursor fibers, the first issue is the solution spinnability. In general, spinnability refers to the ability or the ease of making fibers from a given set of raw materials. ${ }^{2}$ Ide and White $^{3}$ denoted spinnability as the ability to form threads from polymer solutions and melts. Jizheng et $\mathrm{al}^{4}$ defined spinnability as the deformability of a spinning solution under stable stretching force. According to the literature, ${ }^{2,4}$ the allowable maximum takeup velocity of the first winding roller $V_{1 m}$ can be

Correspondence to: D. Pan (dingpan@dhu.edu.cn).

Contract grant sponsor: National Basic Research Program (973 Program); contract grant number: 2006CB606505.

Contract grant sponsor: National Natural Foundation of China; contract grant number: 50333050.

Contract grant sponsor: Shanghai Fundamental Theory Program; contract grant number: 07DJ14002.

Contract grant sponsor: Specialized Research Fund for the Doctoral Program of Higher Education; contract grant number: 20020255010.

Journal of Applied Polymer Science, Vol. 110, 1997-2000 (2008) (C) 2008 Wiley Periodicals, Inc. used to characterize the spinnability; for wet spinning, $V_{1 m}$ was the critical take-up velocity below which the spinning will remain stable. Spinning above this velocity is impossible, for no continuous filament can be obtained. In the case of dry-jet wet spinning, we define $V_{1 m}$ as the critical take-up velocity of the first winding roller beyond which the asspun fiber in the air gap (before entering the coagulating bath) will break.

Theoretically, spinnability depends on many spinning variables, including the rheological properties of the liquid to be spun, spinning temperature, jet stretch, the spinneret hole size and shape, the rate of mass, and heat transfer between the extruded liquid flow, and the coagulation medium and coagulation conditions. ${ }^{5,6}$ Studies on the influences of these factors on the spinnability of various solutions have been reported by many researchers, most of which however were aimed at wet spinning and/or melt spinning. ${ }^{2,3,5}$ In this article, the spinnability of PANDMSO solution in dry-jet wet spinning process and the important factors involved were investigated.

\section{EXPERIMENTAL}

\section{Materials and equipment}

PAN copolymers (acrylonitrile : itaconic acid $=98: 2$ ) were purchased from Shanghai Institute of Synthetic Fiber with a viscosity-average molecular weight 


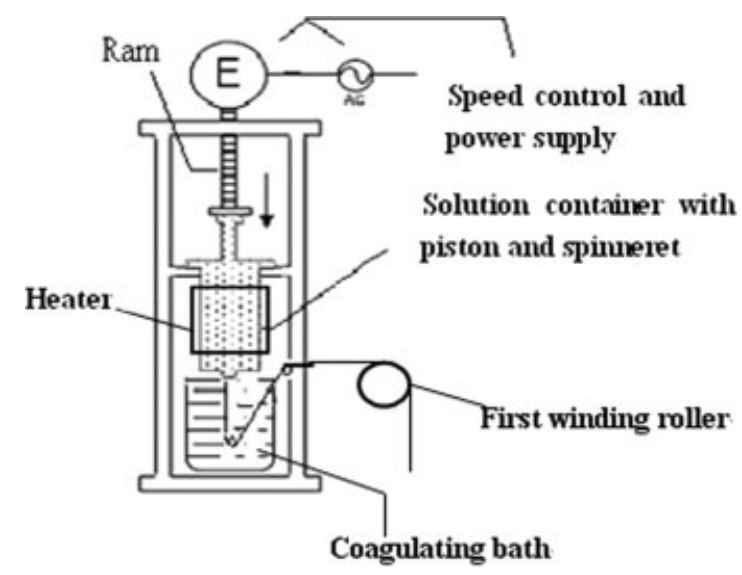

Figure 1 Sketch of the experimental spinning machine.

$\overline{W_{V}}=7.8 \times 10^{4} \mathrm{~g} \mathrm{~mol}^{-1}$. DMSO (analytically pure) was purchased from Shanghai Wulian Chemical Industry, and deionized water was used as the nonsolvent.

A sketch of a piston-type spinning machine custom made to fabricate PAN precursor fibers is shown in Figure 1. A precisely controlled heater was attached to the solution container to adjust the spinning temperature accurately. The coagulating bath temperature was also controlled by a thermostat. The two small rollers in and near the coagulating bath were both passive and made of polished stainless steel. They are guide rollers to assure a smooth movement of the spun thread. Whereas the first winding roller in the figure was active whose rotational speed was adjustable via a motor. It was made of nylon 6 with the surface appropriately roughened to avoid slippage of the spun thread. In addition, the as-spun fiber was still sticky enough that the slippage during winding is negligible. It is thus clear that the maximum take-up velocity of the first winding roller $V_{1 m}$ is equivalent to the linear velocity of the filament.

\section{Experimental conditions}

Spinning solutions of different PAN concentrations by weight $(17,20$, and $23 \%$ ) were prepared with DMSO as the solvent. The ones with 20 wt $\%$ PAN were then added to different amount of water $(0,2$, 3,4 , and 5 wt \%). The coagulating baths with concentrations of $0,25,50$, and $75 \%$ DMSO by weight were prepared, of which the one with $50 \mathrm{wt} \%$ DMSO was used as the control in this work. The spinneret fills with individual holes of the length-diameter ratio 10 and diameter $0.8 \mathrm{~mm}$. The velocity of freely extruded solution was $3.3 \mathrm{~m} / \mathrm{min}$.

\section{Measurement}

The rotational speed of the first winding roller was measured and $V_{1 m}$ determined as $V_{1 m}=\pi D \cdot \omega_{m}$,

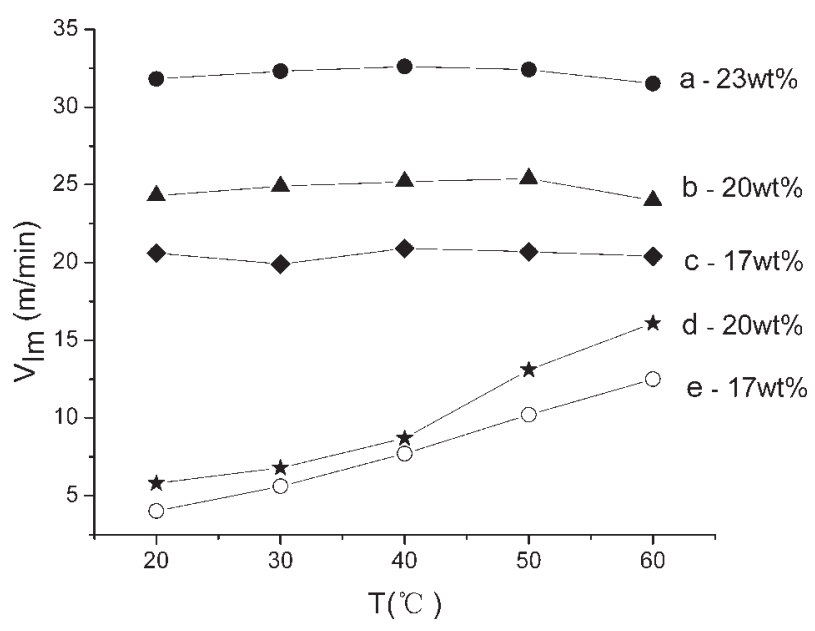

Figure 2 Relations between the coagulating bath temperature $T$ and $V_{1 m}$. The spinning temperature was $70^{\circ} \mathrm{C}$ and the coagulating bath concentration was $50 \mathrm{wt} \%$, air gap length $30 \mathrm{~mm}$ (for dry-jet wet spinning).

where $D$ is the diameter of the roller and $\omega_{m}$ is its maximum rotational speed.

\section{RESULTS AND DISCUSSION}

\section{Influence of the coagulation conditions on} spinnability

Figures 2 and 3 show that both the temperature and concentration of the coagulating bath exert little influence on spinnability $\left(V_{1 m}\right)$ in the case of dry-jet wet spinning (curves $a, b$, and c), unlike in wet spinning where $V_{1 m}$ is greatly affected by these two conditions (curves $\mathrm{d}$ and e).

For wet spinning, the fluid flow coagulates upon it emerges from the spinneret hole. The viscoelastic fluid streams will break if the deformation rate

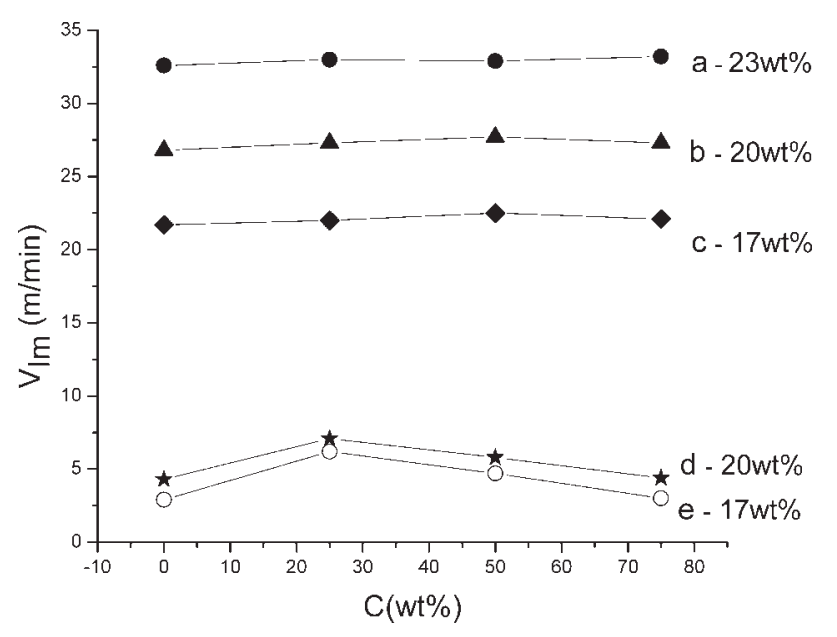

Figure 3 Relations between the coagulating bath concentration $C$ and $V_{1 m}$. The spinning temperature was $70^{\circ} \mathrm{C}$, the coagulating bath temperature $20^{\circ} \mathrm{C}$, air gap length 30 $\mathrm{mm}$ (for dry-jet wet spinning). 
exceeds a critical value, thus $V_{1 m}$ is defined and restricted. We can see from Figure $2(\mathrm{~d}, \mathrm{e})$ that $V_{1 m}$ increases with the coagulating bath temperature, for higher temperature accelerates the mutual diffusion between the fluid flow and the coagulating bath, resulting in quicker solidification. Also in Figure $3(\mathrm{~d}, \mathrm{e}), V_{1 m}$ increases with the coagulating bath concentration when the concentration is below $25 \mathrm{wt} \%$. When the concentration is higher, however, $V_{1 m}$ begins to decrease. This can also be explained by the mutual diffusion mechanism.

For dry-jet wet spinning, the fluid streams will not break as easily even at large deformation rate because of the existence of the dry air gap before entering the coagulating bath. The breakage of the viscoelastic fluid jets is the type of cohesive failure resulted from excessive storage of elastic energy. ${ }^{7}$ This will occur once a critical stress level, expressed by eq. (1), is reached: $:^{3,8}$

$$
\sigma_{\mathrm{c}}=\sqrt{2 K E}
$$

where $\sigma_{c}$ is the critical stress applied to the fluid jets, $K$ and $E$ are the cohesive energy density and the tensile modulus of the fluid flow, respectively. The existence of air gap helps to improve spinnability in that an air gap in appropriate length favors the stress relaxation of the fluid flow as well as the orientation of the PAN molecules. $V_{1 m}$ in dry-jet wet spinning is hence much larger than that in wet spinning, indicating a much better spinnability. Because breakage of the fluid jets always occurs in the air gap, the spinnability $\left(V_{1 m}\right)$ is not affected by the coagulation conditions, as illustrated clearly in Figures 2 and 3.

Another intriguing phenomenon demonstrated by Figures 2 and 3 is that the influence of the concentration of the spinning solution on $V_{1 m}$ is greater in dry-jet wet spinning than in wet spinning. The likely mechanisms are as follows. For wet spinning, the die swell and the quickly formed skin-core structure of the fluid flow when it enters the coagulating bath are the main factors affecting the spinnability. In the data range of this article, the effect of concentration is minor compared with that of the two factors above. For the dry-jet wet spinning, however, the effects of both die swell and the skin-core structure are greatly reduced due to the existence of the air gap. Also, the increased concentration enhances the interaction between PAN molecules and thus promotes the aggregation. Therefore, the spinnability represented by $V_{1 m}$ is significantly improved in the dry-jet wet spinning process.

\section{Influence of the air gap length on spinnability}

The relations between the spinnability $V_{1 m}$ and the air gap length $d$ in dry-jet wet spinning were also

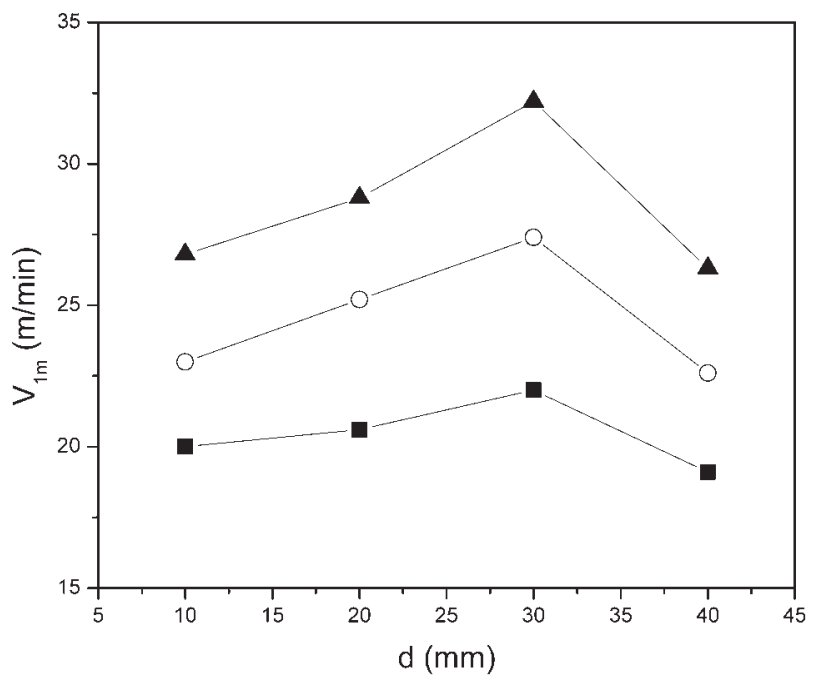

Figure 4 Relations between $V_{1 m}$ and the air gap length $d$. The concentration of spinning solution: $\mathbf{\square - 1 7} \mathrm{wt} \%, \bigcirc-$ 20 wt $\%$, and $\boldsymbol{\Delta}-23$ wt $\%$. The coagulating bath concentration and temperature were $50 \mathrm{wt} \%$ and $20^{\circ} \mathrm{C}$, respectively. The spinning temperature was $70^{\circ} \mathrm{C}$.

examined in this study as shown in Figure 4. For given spinning solutions, $V_{1 m}$ goes up with the increase of $d$ from 10 to $30 \mathrm{~mm}$ but decreases when $d$ is further raised to $40 \mathrm{~mm}$. The initial increase of $V_{1 m}$ is ascribed to the air gap, which facilitates the stress relaxation and makes the cohesive failure more difficult to occur, as mentioned earlier. Nevertheless, once the air gap length exceeds $30 \mathrm{~mm}$, the effect from the capillarity, causing the surface tension-induced breakage of fluid streams, becomes the dominant fracture mechanism. Ziabicki ${ }^{8}$ studied the mechanism of capillarity, and the disturbance propagated by the capillarity can be represented by the amplitude of capillary waves:

$$
\delta=\delta_{0} \exp (\mu t) \cos (2 x / \lambda)
$$

where $\delta_{0}$ is the initial amplitude of capillary waves, $\mu$ the growth factor of capillary waves, $t$ the time, $x$ the distance from the spinneret, and $\lambda$ the wavelength. If the amplitude $\delta$ increases to the same value as the radius of the unperturbed fluid jets, the fluid jets will break down. Increased air gap length will prolong the amplitude of the capillary waves. Therefore, too long an air gap length will render the fluid jets vulnerable as well.

\section{Influence of the nonsolvent content in spinning solution on spinnability}

We can see from our data in Figure 5 that the influence of the water content on $V_{1 m}$ is nonmonotonic. Addition of water into the PAN-DMSO solution weakens the interaction between PAN and the sol- 
vent DMSO whilst strengthens the attraction force between PAN molecular chains, both causing the PAN molecular chains to aggregate and form physical crosslinkings, leading to gelation of the solution. The viscosity of the gelled solution is too large for fiber spinning.

\section{Influence of the spinning temperature on spinnability}

In Figure 6, $V_{1 m}$ rises with the spinning temperature in the range of $65-72{ }^{\circ} \mathrm{C}$ while falls in the range of $72-80^{\circ} \mathrm{C}$, despite the amount of water added in the spinning solution. If we consider the relationship between temperature $\rightarrow$ solution viscosity $\rightarrow$ solution spinnability, the explanation is obvious: the optimal temperature $\rightarrow$ optimal viscosity $\rightarrow$ optimal spinnability, all because of changes in the mobility of molecular chains. Besides, the growing surface tension at too high a temperature tends to break the liquid jets into drops as mentioned earlier. It can be expected that the spinnability would further deteriorate if the spinning temperature exceeds beyond $80^{\circ} \mathrm{C}$.

\section{CONCLUSIONS}

Both the temperature and concentration of the coagulating bath exert little influence on spinnability $\left(V_{1 m}\right)$ in the case of dry-jet wet spinning, unlike in wet spinning where $V_{1 m}$ is greatly affected by these conditions. Also, $V_{1 m}$ in dry-jet wet spinning is much greater than that in wet spinning, because of

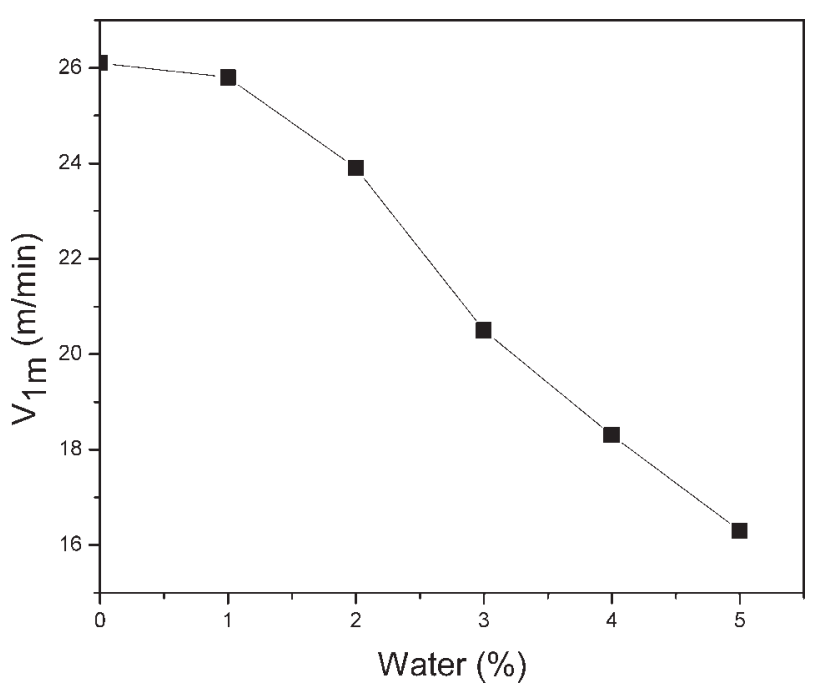

Figure 5 Relation between $V_{1 m}$ and the water content in spinning solution. The coagulating bath concentration and temperature were $50 \mathrm{wt} \%$ and $20^{\circ} \mathrm{C}$, respectively. The concentration of spinning solution was $20 \mathrm{wt} \%$, and the spinning temperature was $70^{\circ} \mathrm{C}$. The air gap length was $30 \mathrm{~mm}$.

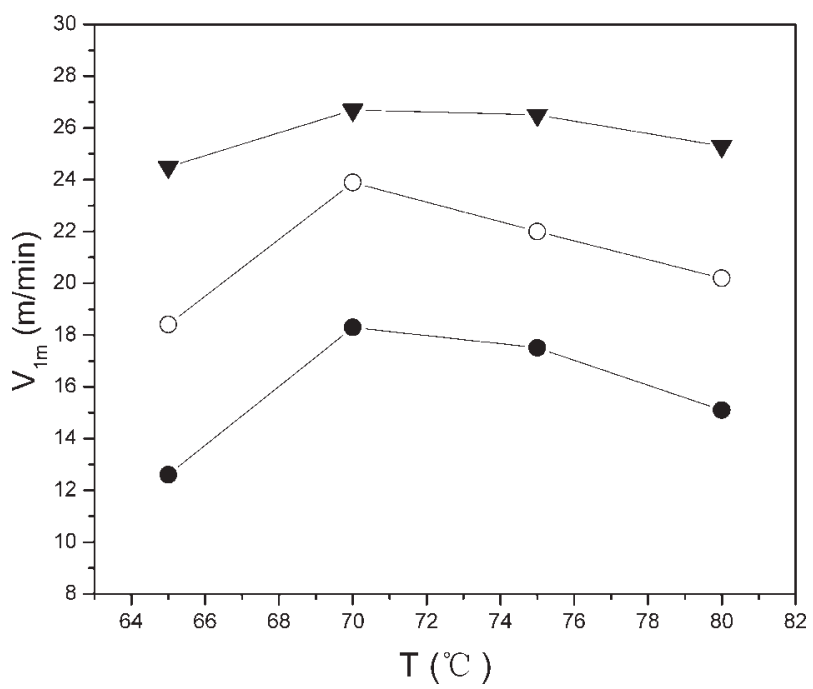

Figure 6 Relations between $V_{1 m}$ and spinning temperature $T$. The water content in spinning solution: $\nabla-0$ wt $\%$, $\mathrm{O}-2 \mathrm{wt} \%$, and $-4 \mathrm{wt} \%$. The concentration of spinning solution was $20 \mathrm{wt} \%$. The coagulating bath concentration and temperature were $50 \mathrm{wt} \%$ and $20^{\circ} \mathrm{C}$, respectively. The air gap length was $30 \mathrm{~mm}$.

the existence of an air gap with appropriate length, which facilitates the spinning process. According to our experimental result, the best air gap length is $30 \mathrm{~mm}$ under the given conditions.

The addition of nonsolvent into the spinning solution, in general, worsens the spinnability, for it adversely alters the rheological properties of the solution. Hence, water should be avoided during the preparation of spinning solution. Spinnability improves with the spinning temperature in the range of $65-72^{\circ} \mathrm{C}$, in which the solution viscosity is most favorable for fiber spinning due to the reduced entanglement of PAN molecular chains and the increased stress relaxation rate in the fluid jets. At higher temperature, the reduced viscosity as well as the rapidly growing surface tension results in breakage of the fluid streams.

\section{References}

1. Serkov, A. T.; Budnitskii, G. A.; Radishevskii, M. B.; Medvedev, V. A.; Zlatoustova, L. A. Fibre Chem 2003, 35, 117.

2. Paul, D. R. J Appl Polym Sci 1968, 12, 2273.

3. Ide, Y.; White, J. L. J Appl Polym Sci 1976, 20, 2511.

4. Jizheng, D.; Honglie, L.; Qingrui, W. Processing Technology of Synthetic Fiber, Vol. 1; Textile Industry Press: Beijing, 1993; pp 180-192.

5. Han, C. D. Rheol Acta 1970, 9, 355.

6. Rahman, M. A.; Ismail, A. F.; Mustafa, A. Mater Sci Eng A Struct 2007, 448, 275.

7. Ziabicki, A. Physics of Fibre Spinning Process; Warszawa: Polish, 1970.

8. Ziabicki, A. Fundamentals of Fibre Formation; Wiley-Interscience: New York, 1976. 